\title{
Cardiac Contractile Dysfunction during Mild Coronary Flow Reductions Is Due to an Altered Calcium-Pressure Relationship in Rat Hearts
}

\author{
Vincent M. Figueredo, Rolf Brandes, Michael W. Weiner, Barry M. Massie, and S. Albert Camacho \\ Cardiovascular Research Institute, the Departments of Medicine (Cardiology) and Radiology, University of California, San Francisco, \\ California 94143; and the Magnetic Resonance Unit, Veterans Administration Medical Center, San Francisco, California 94121
}

\begin{abstract}
Coronary artery stenosis or occlusion results in reduced coronary flow and myocardial contractile depression. At severe flow reductions, increased inorganic phosphate $\left(P_{i}\right)$ and intracellular acidosis clearly play a role in contractile depression. However, during milder flow reductions the mechanism(s) underlying contractile depression are less clear. Previous perfused heart studies demonstrated no change of $\mathrm{P}_{\mathbf{l}}$ or $\mathrm{pH}$ during mild flow reductions, suggesting that changes of intravascular pressure (garden hose effect) may be the mediator of this contractile depression. Others have reported conflicting results regarding another possible mediator of contractility, the cytosolic free calcium $\left(\mathrm{Ca}_{1}\right)$. To examine the respective roles of $\mathrm{Ca}_{1}, \mathbf{P}_{\mathbf{i}}$, $\mathrm{pH}$, and vascular pressure in regulating contractility during mild flow reductions, Indo-1 calcium fluorescence and ${ }^{31} \mathbf{P}$ magnetic resonance spectroscopy measurements were performed on Langendorf-perfused rat hearts. $\mathrm{Ca}_{1}$ and diastolic calcium levels did not change during flow reductions to $50 \%$ of control. $P_{i}$ demonstrated a close relationship with developed pressure and significantly increased from $2.5 \pm 0.3$ to $4.2 \pm 0.4 \mu \mathrm{mol} / \mathrm{g}$ dry weight during a $25 \%$ flow reduction. $\mathrm{pH}$ was unchanged until a $50 \%$ flow reduction. Increasing vascular pressure to superphysiological levels resulted in further increases of developed pressure, with no change in $\mathrm{Ca}_{1}$. These findings are consistent with the hypothesis that during mild coronary flow reductions, contractile depression is mediated by an altered relationship between $\mathrm{Ca}_{1}$ and pressure, rather than by decreased $\mathrm{Ca}_{1}$. Furthermore, increased $P_{1}$ and decreased intravascular pressure may be responsible for this altered calcium-pressure relationship during mild coronary flow reductions. (J. Clin. Invest. 1992. 90:1794-1802.) Key words: inorganic phosphate $\bullet$ pH $\bullet$ contractility $\bullet$ ischemia $\bullet$ energy metabolism

\section{Introduction}

Although myocardial contractility is very sensitive to reductions in blood flow due to coronary artery stenosis or occlusion, the mechanisms responsible for this contractile depression are not completely understood. Factors thought to play a causal role in ischemic contractile dysfunction are $(a)$ a decrease of

Preliminary results of this study were presented at the 64th Scientific Session of the American Heart Association, Anaheim, CA, 11-14 November 1991, and the 41st Annual Scientific Session of the American College of Cardiology, Dallas, TX, 12-16 April 1992.

Address reprint requests to Vincent M. Figueredo, M.D., Magnetic Resonance Unit, Veterans Administration Medical Center, $4150 \mathrm{Cle-}$ ment Street (11M), San Francisco, CA 94121.

Received for publication 21 October 1991 and in revised form 20 May 1992.

The Journal of Clinical Investigation, Inc.

Volume 90, November 1992, 1794-1802 the cytosolic free calcium transient, $(b)$ an accumulation of intracellular inorganic phosphate $\left(\mathrm{P}_{\mathbf{i}}\right)^{1}$ or $\mathrm{H}^{+},(c)$ energetic failure due to ATP depletion or a decrease of the free energy change of ATP hydrolysis $\left(\Delta \mathrm{G}_{\mathrm{ATP}}\right)$, and $(d)$ a decrease of intravascular pressure (garden hose effect). The relative importance of these factors may vary depending on the severity of coronary flow reduction.

To assess the role of cytosolic free calcium in depressing contractility during reductions of coronary flow, several investigators have measured the cytosolic free calcium transient during ischemia (1-4). However, these studies have yielded conflicting results. Lee et al. (1), using the calcium fluorescent probe Indo-1, and Kihara et al. (2), using the bioluminescent calcium indicator aequorin, both showed an acute increase of the calcium transient as contractility decreased during no-flow ischemia. In contrast, Wikman-Coffelt et al. (3), also using Indo-1, reported a $21 \%$ decrease of the calcium transient during a reduction of perfusion pressure from 110 to $80 \mathrm{mmHg}$. Furthermore, Kitakaze and Marban (4), using the fluorine magnetic resonance spectroscopy calcium indicator 5,5'-difluoro derivative of 1,2-bis-(o-aminophenoxy)ethane- $N, N$, $N^{\prime}, N^{\prime}$-tetraacetic acid (5F-BAPTA), demonstrated a $>50 \%$ decrease of the calcium transient during a $20 \%$ reduction of coronary flow. Thus, the relationship of cytosolic free calcium to contractility during reduced coronary flow remains controversial.

Other investigators have examined the roles of intracellular acidosis and alterations of energy phosphate metabolism in depressing contractility during coronary flow reductions. Several studies have unequivocally demonstrated increases of $\mathbf{P}_{i}$ and $\mathrm{H}^{+}$and a decrease of $\Delta \mathrm{G}_{\mathrm{ATP}}$ (5-9) after reductions of coronary flow below $50 \%$ of control. However, greater controversy exists as to the mechanism(s) underlying the fall of contractility associated with milder coronary flow reductions (i.e., 50-99\% of control). In in vivo animal models, changes of energy phosphate metabolites have been demonstrated when coronary flow or perfusion pressure was reduced to 70 to $75 \%$ of control $(10,11)$. Although the increase of $P_{i}$ was relatively small at this level of coronary flow reduction, it was of a magnitude that has been associated with marked reductions of force and an alteration of the calcium-force relationship in skinned myofiber studies $(12,13)$. In contrast, previous perfused heart studies $(6,7,14,15)$ reported no change in energy phosphates as measured by ${ }^{31} \mathrm{P}$ magnetic resonance spectroscopy $\left({ }^{31} \mathrm{P}\right.$ MRS) during mild coronary flow reductions (i.e., $50-99 \%$ of

1. Abbreviations used in this paper: $\Delta \mathrm{Ca}_{\mathrm{i}}$, cytosolic free calcium transient amplitude; $\mathrm{dCa}_{\mathrm{i}}$, diastolic calcium; F385/F454, ration of Indo-1 fluorescence intensities at emission wavelengths of 385 and $454 \mathrm{~nm}$; 5F-BAPTA, 5,5'-difluoro derivative of 1,2-bis-( 0 -aminophenoxy)ethane- $N, N, N^{\prime}, N^{\prime}$-tetraacetic acid; MPA, methylphosphonic acid; $\mathrm{P}_{\mathrm{i}}$, inorganic phosphate; ${ }^{31} \mathrm{P}$-MRS, ${ }^{31} \mathrm{P}$ magnetic resonance spectroscopy. 
baseline flow). This would suggest that a factor(s) other than $\mathbf{P}_{i}$ and $\mathrm{H}^{+}$, such as decreased intravascular pressure, mediates contractile dysfunction during mild coronary flow reductions. However, technical factors, such as an inadequate signal/noise ratio of the ${ }^{31} \mathrm{P}$-MRS spectra and experimental design, may have made it difficult to measure small changes of $\mathrm{P}_{\mathrm{i}}$ and $\mathrm{H}^{+}$.

Therefore, to clarify the mechanism(s) underlying the myocardial contractile depression associated with mild reductions of coronary flow, the present study was designed to test the following hypothesis: during mild coronary flow reductions, contractile depression results from an altered relationship between cytosolic free calcium and pressure rather than by a decrease of the cytosolic free calcium transient amplitude. Additionally, parallel experiments were performed to determine whether this alteration of the cytosolic free calcium-pressure relationship might be due to increased $\mathrm{P}_{\mathrm{i}}, \mathrm{H}^{+}$, and/or decreased intravascular pressure. Indo- 1 calcium fluorescence was used as an index of cytosolic free calcium and ${ }^{31} \mathrm{P}$-MRS was used to measure $\mathrm{P}_{\mathrm{i}}$ and $\mathrm{H}^{+}$in parallel isolated rat heart experiments.

\section{Methods}

\section{Heart perfusion and measurement of function}

Male Sprague-Dawley rats (450-550 g) were heparinized using 1,000 $\mathrm{U}$ i.p. and anesthetized using ketamine $100 \mathrm{mg}$ i.p. Hearts were then excised and immediately arrested in cold isosmotic saline containing $20 \mathrm{mmol} /$ liter $\mathrm{KCl}$. Isolated hearts were perfused retrograde via the aorta at a constant pressure of $71 \mathrm{mmHg}$, using a Krebs-Henseleit perfusate containing the following (mmol/liter): $118 \mathrm{NaCl}, 6.0 \mathrm{KCl}$, $2.5 \mathrm{CaCl}_{2}, 25 \mathrm{NaHCO}_{3}, 1.2 \mathrm{MgSO}_{4}, 0.5 \mathrm{Na}_{2} \mathrm{EDTA}, 4.0$ glucose, 10.0 pyruvate, and $20 \mathrm{U} /$ liter insulin. The perfusate was continuously bubbled using a $95 \% \mathrm{O}_{2} / 5 \% \mathrm{CO}_{2}$ gas mixture and maintained at $37^{\circ} \mathrm{C}$. A compliant latex balloon attached to a Trantec pressure transducer (American Edwards Laboratories, Irvine, CA) via rigid polyethylene tubing was inserted into the left ventricle to measure pressure. Left ventricular diastolic pressure was set at $5-10 \mathrm{mmHg}$ by adjusting the balloon volume. Developed pressure was recorded on a chart recorder (series 8000; Gould Electronics, Hayward, CA). Hearts were paced at 300 beats/min using two platinum tipped electrodes connected to a stimulus generator (SD-5; Grass Instrument Co., Quincy, MA). Coronary flow was measured by collecting the heart effluent. Coronary flow reductions were produced using an in-line flowmeter that controlled coronary perfusion pressure (Gilmont Instruments, Barrington, IL). In a separate series of experiments, perfusion and developed pressures were measured over a range of coronary flows (50-140\% of control), using a $\mathrm{T}$ connection and Trantec pressure transducer placed at the level of the heart ( $n=5$ for $50-100 \% ; n=4$ for $100-140 \%$; see Fig. 1 ).

\section{Indo-1 calcium fluorescence measurements}

Fluorescence instrumentation. Fluorescence studies were performed using a modified spectrofluorometer (48000S; SLM Instruments, Inc., Urbana, IL). Excitation light from a 450-W xenon arclamp (SLM Instruments, Inc.) was filtered through a $360-\mathrm{nm}$ interference filter and focused onto the in-going leg of a quartz bifurcated fiber bundle. The common leg of this $1.57-\mathrm{mm}$ diameter fiber bundle was girdled against the epicardial surface of the left ventricle, avoiding visible vessels. A shutter in front of the excitation light was opened for only seconds at a time during data acquisition to prevent bleaching of the Indo-1 fluorescence. The fluorescent signal was transferred via the out-going leg of the bundle and separated by a dichroic mirror onto two photomultiplier tubes preceeded by 385 and 454-nm interference filters.

Indo-1 methods. After a 30-min equilibration period, NADH background fluorescence measurements ( see Calcium transient determina- tion below) were obtained. Hearts were then loaded with the cell-permeable fluorescent calcium indicator Indo-1 AM (Molecular Probes, Inc., Junction City, OR). Hearts were loaded for 30 to $45 \mathrm{~min}$ by perfusion with $150 \mathrm{ml}$ Krebs-Henseleit buffer containing $5 \mu \mathrm{M}$ Indo-1 AM dissolved with Pluronic F-127 in dimethylsulfoxide (20\% wt/vol; $1.5 \mathrm{ml}$ ) in the presence of $5 \%$ fetal calf serum. Residual Indo-1 AM was washed out by perfusing with standard buffer for $30 \mathrm{~min}$. Loading with Indo-1 resulted in a 13- to 27-fold increase in fluorescence at the 385nm emission wavelength compared with baseline background fluorescence and a 5-25\% depression of developed pressure. Probenecid $(0.1$ $\mathrm{mM}$ ) was added to all buffer solutions to slow the extrusion of Indo-1 from myocytes. Using this technique, high signal-to-noise fluorescence signals were present for $>2 \mathrm{~h}$.

Calcium transient determination. Cytosolic free calcium was monitored using the ratio of Indo-1 fluorescence intensities at emission wavelengths of 385 and $454 \mathrm{~nm}$ (F385/F454). Three factors that could affect cytosolic free calcium determination were taken into account: motion artifact; tissue inner filter effect, which is a consequence of the myoglobin oxygenation state; and changes of heart background fluorescence, which is primarily NADH $(16,17)$. Motion artifact was minimized by securing the fiber bundle to the heart using a nylon girdle and by monitoring two emission wavelengths that are relatively close in the fluorescence spectrum, as this minimizes differences in motion artifact at different wavelengths (16). Changes in tissue filter absorption (predominantly due to myoglobin) were minimized by using emission wavelengths that have a constant absorbance relative to changes of the tissue oxygenation state $(16,17)$. Changes of NADH background fluorescence during coronary flow reductions were accounted for by measuring the NADH background fluorescence before Indo-1 loading at control flow and during a flow reduction identical to that performed after Indo-1 loading ( see Experimental protocol below). These NADH background fluorescence values were then subtracted, using SLM software (SLM Instruments, Inc.) from the appropriate control or low flow Indo-1 fluorescence spectrum at each emission wavelength (16). F385/F454 was then calculated using the corrected F385 and F454 intensities and used as an index of the cytosolic free calcium transient. Relative changes of the cytosolic free calcium transient amplitude $\left(\Delta \mathrm{Ca}_{\mathrm{i}}\right)$ were determined by measuring the difference between the maximum fluorescence intensity of F385/F454 and the minimum fluorescence intensity of F385/F454 at baseline and during each coronary flow reduction. Relative changes of diastolic calcium $\left(\mathrm{dCa}_{\mathrm{i}}\right)$ were determined by measuring the minimum fluorescence intensity of $\mathrm{F} 385$ / F454 at baseline and during each coronary flow reduction.

Bradykinin effect. A previous study by Lorell et al. (18) demonstrated a marked increase of the Indo-1 fluorescence signal in rabbit hearts after infusion of bradykinin, most likely due to significant Indo1 fluorescence from endothelial cells. Therefore, studies were performed comparing Indo-1 calcium fluorescence measurements before and during infusion with bradykinin $\left(10^{-5} \mathrm{M}, n=2\right)$ to determine if bradykinin increased the Indo-1 fluorescence signal in rat hearts.

Sensitivity of Indo-1 calcium fluorescence technique. To assess the sensitivity of the Indo-1 calcium fluorescence technique for demonstrating changes of cytosolic free calcium, experiments were performed in which perfusate $\left[\mathrm{Ca}^{2+}\right]$ was varied between 1.00 and $2.50 \mathrm{mM}$ after loading of Indo- 1 . Relative changes of $\Delta \mathrm{Ca}_{\mathrm{i}}$ were determined by measuring changes in F385/F454.

Transmural studies. To rule out the possibility that Indo- 1 calcium fluorescence measurements at the subepicardium do not truly represent the sequelae of mild coronary flow reductions across the entire myocardium, additional experiments were performed in which Indo-1 calcium fluorescence measurements were obtained from the subendocardium using the identical experimental protocol $(n=6$; see Experimental protocol below). To obtain subendocardial Indo-1 calcium fluorescence data, a $0.69-\mathrm{mm}$ fiber bundle was passed through the left atrium and mitral valve onto the septal endocardial surface of the left ventricle adjacent to the left ventricular pressure balloon. Subendocardial data was analyzed in the same manner as subepicardial data. 
NADH measurements. To confirm that changes of NADH during mild flow reductions were stable over the 10-min data acquisition periods (after 2.5-min equilibration periods; see Experimental protocol below) experiments were performed in unloaded hearts in which coronary flow was reduced to 50 and $75 \%$ of control for $12.5 \mathrm{~min}(n=10)$. Control measurements of heart background fluorescence (primarily $\mathrm{NADH}$ ) were compared with measurements taken at 2.5 and $12.5 \mathrm{~min}$ after a flow reduction and after a 10 -min recovery period at control flow. Repeated measurements in the same heart were performed to assess the reproducibility of background fluorescence changes.

\section{${ }^{31} P$-MRS acquisition measurements}

${ }^{31}$ P-MRS data were acquired using a 7 Tesla horizontal $18 \mathrm{~cm}$ bore magnet MRI/MRS system (Nalorac Cryogenics Corp., Martinez, CA) using a custom-built probe that placed the perfused heart within a 25-mm diameter solenoid coil. Transients were collected after the application of $18^{\circ}$ pulses every $0.1 \mathrm{~s}$. A total of 1,200 transients were collected over 2 min and summed into $4 \mathrm{~K}$ blocks. Five consecutive 2-min spectra were collected over 10-min acquisition periods, and then summed into 10-min blocks to improve the signal/noise ratio and determine $P_{i}, P C r$, and $\beta$-ATP concentrations (ATP). To avoid subjective errors in manual frequency domain fitting of the MRS spectra, an automated fitting program using the variable projection method (19) was used to determine peak areas and chemical shifts. Intracellular $\mathrm{pH}$ was calculated from the chemical shift of $P_{i}$ relative to $\mathrm{PCr}$ (5). Spin lattice relaxation times $\left(T_{1}\right)$ were measured to correct for saturation effects. Absolute concentrations of metabolites were calculated by the method described by Humphrey and Garlick (20) using a methylphosphonic acid (MPA) standard. Briefly, a capillary tube containing a solution of MPA ( $1.590 \mathrm{mM})$ and $\mathrm{MnCl}_{2}(16 \mu \mathrm{M}$, included to allow complete relaxation of MPA signal at a repetition rate of $0.1 \mathrm{~s}$ ) was fixed inside the solenoid coil. The amount of MPA detected by the coil was determined by comparison with a phantom containing $P_{i}(2 \mathrm{mM})$ diluted in Krebs-Henseleit perfusate and placed in the same position as hearts within the limits of the coil. The phantom was surrounded by perfusate to mimic the perfused heart setup. 480 spectra were accumulated using $90^{\circ}$ pulses with a repetition time of $120 \mathrm{~s}$. Quantitation of $\mathbf{P}_{i}, \mathbf{P C r}$, and ATP relative to the corrected MPA standard content was then performed and expressed in micromoles per gram-dry-weight ( $\mu \mathrm{mol} / \mathrm{g}$ dry wt $). \Delta \mathrm{G}_{\mathrm{ATP}}$ was calculated from the creatine kinase equilibrium by converting $\mu \mathrm{mol} / \mathrm{g}$ dry wt data to millimolar concentrations (assuming an intracellular water content of $0.44 \mathrm{ml} / \mathrm{g}$ wet wt) and using the equation: $\Delta_{\mathrm{ATP}}=\Delta \mathrm{G}^{\circ}+\mathrm{RT} \ln \left([\mathrm{Cr}]\left[\mathrm{P}_{\mathrm{i}}\right] /[\mathrm{PCr}]\left[\mathrm{H}^{+}\right] K_{\mathrm{eq}}\right)$ where $\Delta \mathrm{G}^{\circ}=-30.5 \mathrm{~kJ} / \mathrm{mol}, K_{\mathrm{eq}}=10^{9} \mathrm{M}^{-1}$ and $[\mathrm{Cr}]=(25.44 \mathrm{mM}$ $-[\mathrm{PCr}])(21,22)$.

\section{Experimental protocol}

Parallel experimental protocols were used for the Indo-1 calcium fluorescence and ${ }^{31} \mathrm{P}$-MRS experiments. After a 30-min equilibration period, control measurements were taken of developed pressure, coro- nary flow, and $\Delta \mathrm{Ca}_{\mathrm{i}}$ and $\mathrm{dCa}_{\mathrm{i}}$ (after Indo-1 loading) or energy phosphates. This was followed by a 12.5 -min period of a coronary flow reduction ( 50 or $75 \%$ of control in the Indo-1 protocol; $50,65,75$, or $85 \%$ of control in the ${ }^{31} \mathrm{P}$-MRS protocol). No measurements were taken during the initial $2.5 \mathrm{~min}$ of ischemia to allow developed pressure, fluorescence intensities, and energy phosphates to equilibrate (unpublished observations from this laboratory). Data were acquired during the subsequent 10 -min period. To confirm that a steady state had been reached over the 10-min acquisition period, levels of developed pressure, NADH (before Indo-1 loading), $\Delta \mathrm{Ca}_{\mathrm{i}}$ and $\mathrm{dCa}_{\mathrm{i}}$, and $\mathrm{PCr}$ and ATP ( first and last $2 \mathrm{~min}{ }^{31} \mathrm{P}$-MRS spectra) were measured at 2.5 and 12.5 min of each coronary flow reduction. ${ }^{31} \mathrm{P}-\mathrm{MRS}$ measurements were performed at three to five different coronary flow reductions in random order $(n=11)$. A 10-min recovery period followed each flow reduction, which allowed developed pressure and coronary flow to return to baseline levels. One or two coronary flow reductions were studied during the Indo-1 protocol ( $n=8$ at a coronary flow of $50 \%$ of control, $n=4$ at a coronary flow of $75 \%$ of control), with one second fluorescence spectra acquired after the 2.5 -min equilibration period and again at $12.5 \mathrm{~min}$ of a coronary flow reduction to confirm that there was no change of F385/F454 during the 10-min period required to obtain high signal-to-noise ${ }^{31} \mathrm{P}-\mathrm{MRS}$ data. At the end of each experiment a set of control measurements were taken to confirm there were no changes from the initial control measurements.

In a separate series of experiments, cytosolic free calcium, coronary flow, and developed pressure were measured at superphysiological perfusion pressures (72-95 $\mathrm{mmHg}$ ). ${ }^{31} \mathrm{P}-\mathrm{MRS}$ measurements were not acquired at these elevated perfusion pressures because at the physiological control perfusion pressure $\left(72 \mathrm{mmHg}\right.$ ) the $P_{i}$ concentration is already at the limits of resolution, even with the improved signal to noise of the present ${ }^{31} \mathrm{P}$-MRS studies. Therefore, a further decrease of $P_{i}$ (if it exists) could not be detected at superphysiological perfusion pressures.

\section{Statistical analyses}

Values are reported as mean \pm SEM. Statistical analyses were performed using paired $t$ tests, repeated measures analysis of variance, or linear regression, when indicated. Results were considered significant when $P$ $<0.05$.

\section{Results}

Hemodynamics. Fig. 1 A graphically represents the relationship between coronary flow and developed pressure over a range of coronary flows from 50 to $140 \%$ of control (baseline flow $=15.1 \pm 0.6 \mathrm{ml} / \mathrm{g}$ wet $\mathrm{wt} \mathrm{min}^{-1}$ ). Control coronary flow was considered to be the coronary flow at the physiological perfusion pressure of $71 \mathrm{mmHg}$ (23). Fig. $1 \mathrm{~B}$ demonstrates a linear relationship between coronary flow and perfusion pressure over the same range. After a 2.5 -min equilibration period,
A

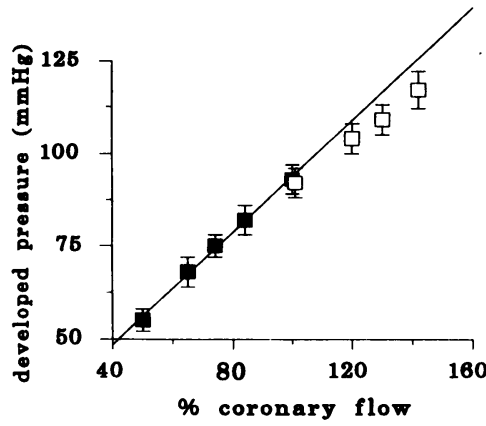

B

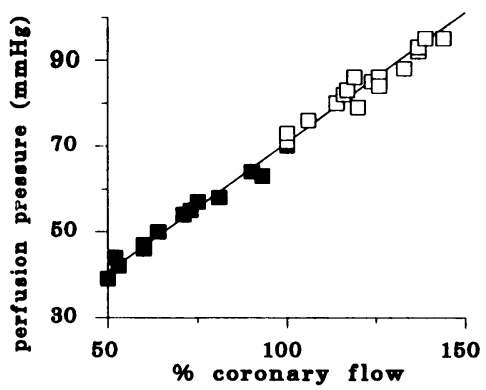

Figure 1. Relationship between $(A)$ coronary flow and developed pressure and $(B)$ coronary flow and perfusion pressure in Langendorf-perfused rat hearts. Coronary flow is expressed as percent of control (coronary flow at the normal physiological perfusion pressure of $71 \mathrm{mmHg}$ ). Data acquired during coronary flow reductions below control levels are denoted with closed squares. Data acquired at superphysiological levels are denoted with open squares. Developed (pooled data) and perfusion (raw data) pressures are expressed in $\mathrm{mmHg}$. Coronary flow demonstrated linear relationships with developed $\left(r^{2}=1.00\right)$ and perfusion $\left(r^{2}=0.98\right)$ pressures during graded coronary

flow reductions. Additional experiments demonstrated that developed pressure continued rising with further increases of perfusion pressure above normal physiological levels, suggesting intravascular pressure is an important mediator of contractile function. 
Table I. Stability of Metabolic and Mechanical Parameters at Steady State during a 50\% Coronary Flow Reduction in Rat Hearts

\begin{tabular}{lccc}
\hline & \multicolumn{3}{c}{ Time } \\
\cline { 2 - 4 } & $0 \mathrm{~min}$ & $2.5 \mathrm{~min}$ & $12.5 \mathrm{~min}$ \\
\hline$\% \mathrm{NADH}$ & 100 & $106 \pm 1^{*}$ & $106 \pm 1^{*}$ \\
PCr & $42.2 \pm 1.3^{\ddagger}$ & $37.8 \pm 1.8^{*}$ & $38.2 \pm 1.9^{*}$ \\
ATP & $15.2 \pm 0.5^{\ddagger}$ & $14.5 \pm 0.7$ & $15.0 \pm 0.8$ \\
Developed pressure & $93 \pm 4$ & $55 \pm 3^{*}$ & $54 \pm 3^{*}$
\end{tabular}

Data from experiments in Langendorf-perfused rat hearts subjected to a $50 \%$ coronary flow reduction for $12.5 \mathrm{~min}$. After a 2.5 -min equilibration period, data were acquired at the beginning and end of a 10min data acquisition period. Changes of NADH background fluorescence are reported as percent of control NADH background fluorescence of a perfused rat heart under normal physiological conditions (\% NADH; $n=10$ ). Phosphocreatine (PCr) and $\beta$-ATP data are expressed as $\mu \mathrm{mol} / \mathrm{g}$ dry wt $(n=11)$ and were calculated from 2-min ${ }^{31} \mathrm{P}$-MRS spectra acquired at control coronary flow and between 2.5-4.5 and 10.5-12.5 min of a 50\% coronary flow reduction. Developed pressures are expressed as $\mathrm{mm} \mathrm{Hg}$. These data demonstrate that functional and metabolic parameters did not change during the 10 min data acquisition period, suggesting a steady state had been reached. Data are mean \pm SEM. ${ }^{*} P<0.05$ versus control values. ${ }^{\ddagger}$ Control values taken immediately before each coronary flow reduction to $50 \%$ of control during graded flow reduction experiments.

developed pressure remained constant over the 10 -min data acquisition period at coronary flow reductions down to $50 \%$ of control ( Table I). To insure data were not altered by myocardial damage or stunning, measurements were repeated under baseline conditions at the end of each experiment. These measurements showed no difference of developed pressure or coronary flow compared with initial control values.

Indo-1 calcium fluorescence results. Fig. 2 shows sample Indo- 1 fluorescence and left ventricular pressure tracings at coronary flows of 100 and $50 \%$ of control. F385/F454 was used as an index of the cytosolic free calcium transient. As shown in Table II, no change of F385/F454 was observed over the range of coronary flow reductions studied, consistent with a lack of change of $\Delta \mathrm{Ca}_{\mathrm{i}}$ or $\mathrm{dCa}_{\mathrm{i}}$ during mild coronary flow reductions. Furthermore, F385/F454 remained constant over the 10-min data acquisition period (necessary to measure $P_{i}$ in ${ }^{31}$ P-MRS experiments) as determined from measurements taken at 2.5 and $\mathbf{1 2 . 5}$ min after each coronary flow reduction, suggesting a steady state of cytosolic free calcium levels had been reached. These results indicate that the fall in myocardial contractility during mild coronary flow reductions is not due to a decrease of the cytosolic free calcium transient. Additional studies demonstrated no change of the cytosolic free calcium transient, despite increases of developed pressure, when perfusion pressure was elevated to superphysiological levels (72-95 $\mathrm{mmHg}$ ).

To determine whether there is a bradykinin-sensitive component to the Indo-1 fluorescence signal, measurements were made before and during infusion with bradykinin $\left(10^{-5} \mathrm{M}\right)$. No significant difference in the Indo-1 calcium fluorescence transient amplitude or diastolic levels was observed, despite an increase of coronary flow (at constant coronary perfusion pressure). This suggests that bradykinin does not significantly affect the Indo-1 calcium fluorescence signal in the perfused rat heart model. This has been previously reported by another laboratory (3).

To determine the sensitivity of the Indo- 1 calcium fluorescence technique for demonstrating relative changes of cytosolic free calcium, experiments were performed in which perfusate $\left[\mathrm{Ca}^{2+}\right]$ was varied between 2.5 and $1.0 \mathrm{mM}$, resulting in varying degrees of decreased contractility. Reducing perfusate $\left[\mathrm{Ca}^{2+}\right.$ ] from 2.5 to $2.0 \mathrm{mM}$ produced a $29 \%$ decrease of developed pressure (comparable to the decrease of developed pressure at a flow reduction to $75 \%$ of control) and a $12 \%$ decrease of the F385/F454 amplitude. A reduction of perfusate $\left[\mathrm{Ca}^{2+}\right]$ from 2.5 to $1.5 \mathrm{mM}$ resulted in a $50 \%$ decrease of developed pressure (comparable to the decrease of developed pressure at a flow reduction to $50 \%$ of control) and a $29 \%$ decrease of the F385/F454 amplitude. These results indicate that the Indo-1 calcium fluorescence technique is sufficiently sensitive to detect changes in cytosolic free calcium that cause alterations in

B

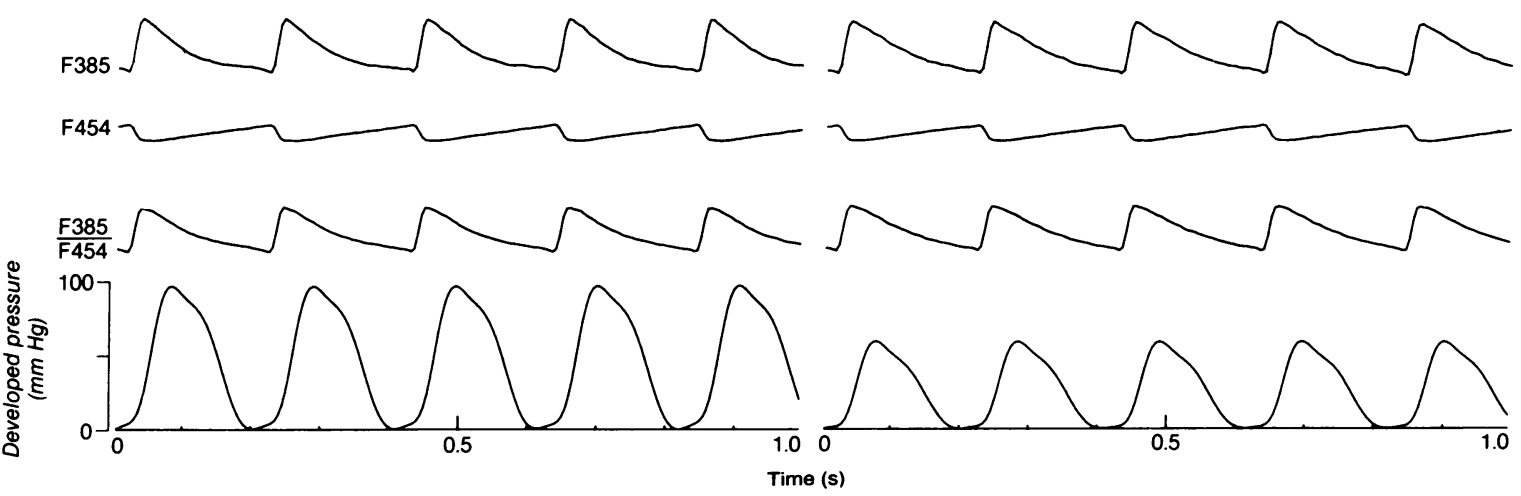

Figure 2. Representative $1 \mathrm{~s}$ Indo-1 calcium fluorescence spectra and developed pressure tracings from Langendorf-perfused rat hearts at $(A)$ control coronary flow and $(B) 50 \%$ of control flow. Tracings from top to bottom show the relative fluorescence intensities of the single emission wavelengths at 385 and $454 \mathrm{~nm}$ ( $F 385$ and $F 454$, respectively), the $385 / 454 \mathrm{~nm}$ ratio $(F 385 / F 454)$, and the developed pressure (mmHg). Indo-1 fluorescence data is presented in arbitrary intensity units. Although a significant fall of developed pressure is observed after a reduction of coronary flow to $50 \%$ of control, no change was observed in $\mathrm{F} 385 / \mathrm{F} 454$, an index of the intracellular cytosolic free calcium transient. 
Table II. Myocardial Hemodynamics, Energy Phosphate Metabolites, and Relative Cytosolic Free Calcium during Mild Coronary Flow Reductions in Rat Hearts

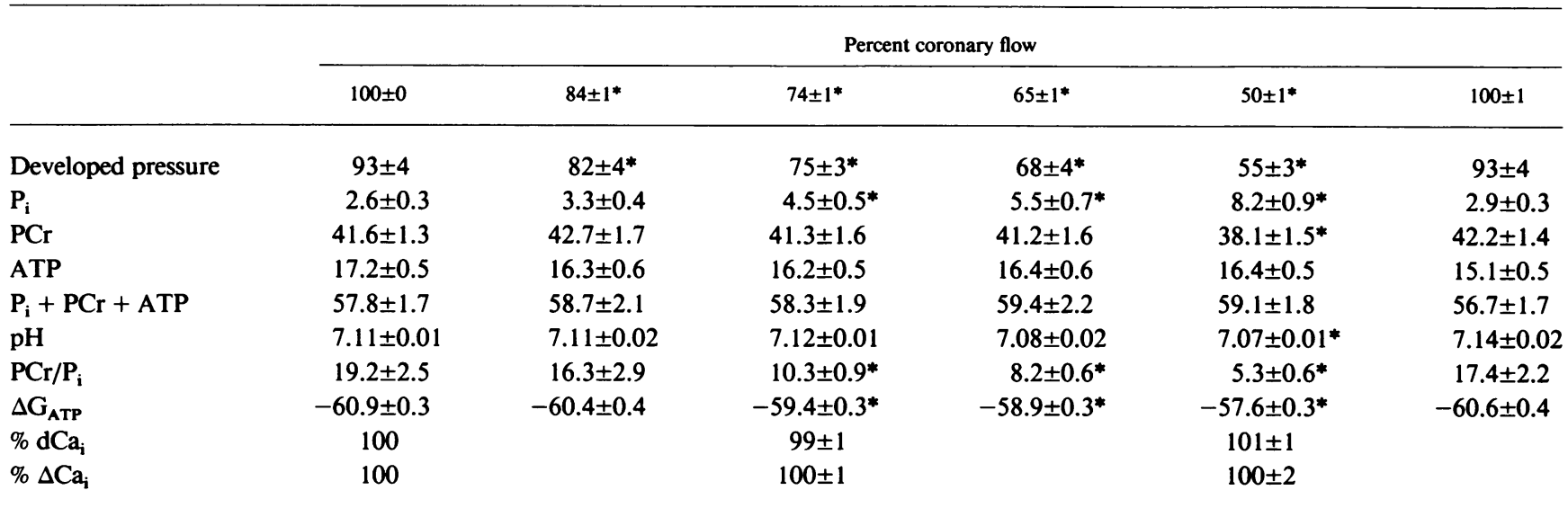

Data from experiments in Langendorf-perfused rat hearts subjected to multiple mild coronary flow reductions. After a 2.5-min equilibration period, data were acquired over 10-min data acquisition periods. 10-min recovery periods separated flow reductions. Coronary flows are expressed as percent of initial baseline flow and developed pressures as $\mathrm{mm} \mathrm{Hg}$. Inorganic phosphate $\left(\mathrm{P}_{\mathrm{i}}\right)$, phosphocreatine $(\mathrm{PCr}), \beta-\mathrm{ATP}$, and $\mathrm{pH}$ were measured using ${ }^{31} \mathrm{P}-\mathrm{MRS}$ and expressed as $\mu \mathrm{mol} / \mathrm{g}$ dry wt $(n=11)$. The $\mathrm{PCr} / \mathrm{P}_{\mathrm{i}}$ ratio $\left(\mathrm{PCr} / \mathrm{P}_{\mathrm{i}}\right)$ and the summed energy phosphate metabolites $\left(P_{i}+P C r+A T P\right)$ were determined from ${ }^{31} P-M R S$ data. The free energy change of ATP hydrolysis $\left(\Delta G_{A T P}\right)$ was calculated from the creatine kinase equilibrium and expressed as $\mathrm{kJ} / \mathrm{mol}$. Relative changes of the cytosolic free calcium transient $\left(\Delta \mathrm{Ca}_{\mathrm{i}}\right)$ and diastolic levels of cytosolic free calcium $\left(\mathrm{dCa}_{\mathrm{i}}\right)$ were determined in a parallel series of experiments using Indo- 1 calcium fluoremetry. Cytosolic free calcium levels were determined by monitoring the ratio of fluorescence intensities at emission wavelengths 385 and $454 \mathrm{~nm}$ and expressed as percent of initial baseline values ( $n=8$ at flow of $50 \%$ baseline, $n=4$ at flow of $75 \%$ baseline). Data are mean \pm SEM. ${ }^{*} P<0.05$.

contractility similar to the contractile depression seen with mild coronary flow reductions in these experiments.

To determine whether the response of F385/F454 to mild coronary flow reductions is similar at the subepicardium and subendocardium, a set of parallel Indo-1 calcium fluorescence experiments were performed on the left ventricular septal endocardial surface. At coronary flow reductions to $50 \%$ of control, no significant change in the amplitude ( $95 \pm 3 \%$ of control ) or diastolic level ( $100 \pm 1 \%$ of control) of F385/F454 was observed. These data suggest that during mild coronary flow reductions to $50 \%$ of control there is no change of cytosolic free calcium at the subepicardium or subendocardium.

Experiments were also performed to assess the severity and stability of NADH background fluorescence changes after mild coronary flow reductions after equilibration of developed pressure and metabolic parameters. After coronary flow reductions to $75 \%$ of control, NADH background fluorescence did not significantly change compared with initial control levels over the 10-min data acquisition period at steady state. However, after coronary flow reductions to $50 \%$ of control, NADH background fluorescence increased $6 \pm 1 \%$ at $2.5 \mathrm{~min}$ and $6 \pm 1 \%$ at 12.5 min of reduced flow compared with initial control values (Table I). Thus, changes of NADH background fluorescence during mild coronary flow reductions remained constant over the 10 -min data acquisition period after a 2.5 -min equilibration period. Multiple flow reductions to $50 \%$ of control flow in the same heart demonstrated these increases in background fluorescence to be reproducible. Control NADH background fluorescence levels after a 10-min recovery period were identical to initial control levels.

${ }^{31} P-M R S$ results. Analysis of the five consecutive 2-min spectra acquired at each coronary flow reduction demonstrated that PCr and ATP remained constant over the 10-min data acquisition periods, confirming that at each flow reduction a steady state had been achieved (Table $I$ ). $P_{i}$ was not quantitated using 2-min spectra due to insufficient signal/noise. Measurements obtained after return to baseline conditions at the end of each experiment demonstrated no change of $\mathrm{P}_{\mathrm{i}}$ or $\mathrm{PCr}$ compared with initial control values. ATP fell modestly over the course of the experiment from $16.2 \pm 0.5$ to $14.2 \pm 0.4 \mu \mathrm{mol} /$ $\mathrm{g}$ dry $w t$, as is usually seen during prolonged isolated heart perfusions (6). The nonspecificity of this change over time relative to the present protocol was confirmed by studying hearts at control coronary flow $(n=2)$. over a time course similar to that in the above experiments. A comparable decrease was noted in ATP, whereas $P_{i}$ and $P C r$ remained constant.

Fig. 3 shows examples of 10-min spectra at coronary flows of 50 and $100 \%$ of control. These spectra demonstrate the high

\section{A Control flow}

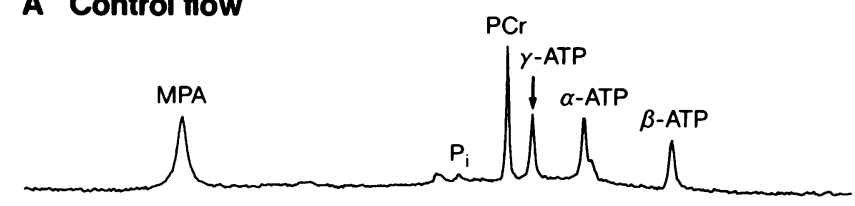

B $\mathbf{5 0 \%}$ control flow

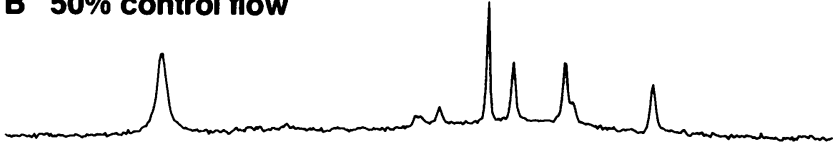

Figure 3. Representative 10-min ${ }^{31} \mathrm{P}-\mathrm{MRS}$ spectra of Langendorfperfused rat hearts at $(A)$ control coronary flow and $(B) 50 \%$ of control flow. $P_{i}$, inorganic phosphate; PCr, phosphocreatine; $\gamma$-ATP, $\alpha$-ATP, and $\beta-A T P$, gamma, alpha, and beta peaks of adenosine triphosphate, $M P A$, methylphosphonic acid reference. Note the increase of the area under the $P_{i}$ peak when coronary flow was reduced to $50 \%$ of control flow. 
a
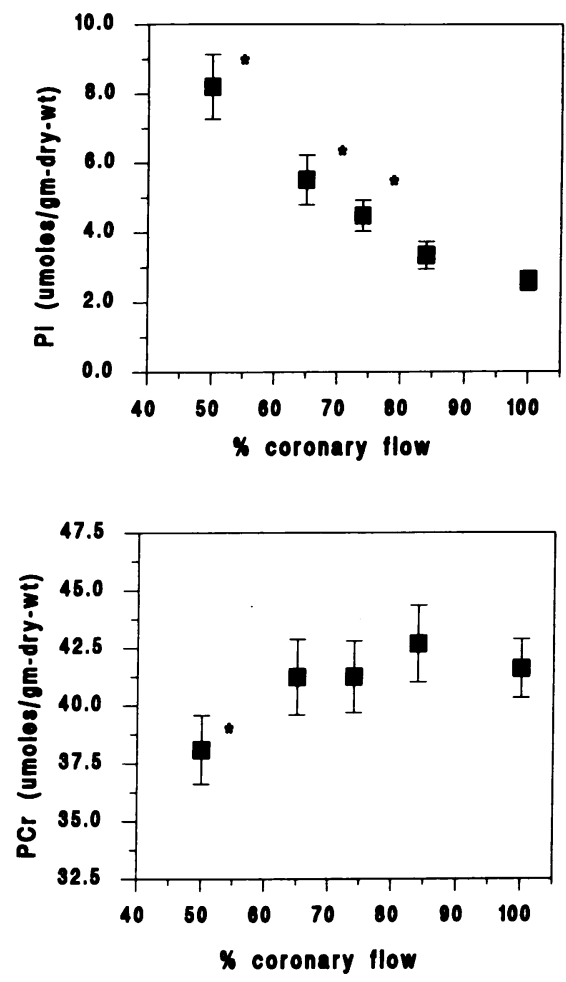

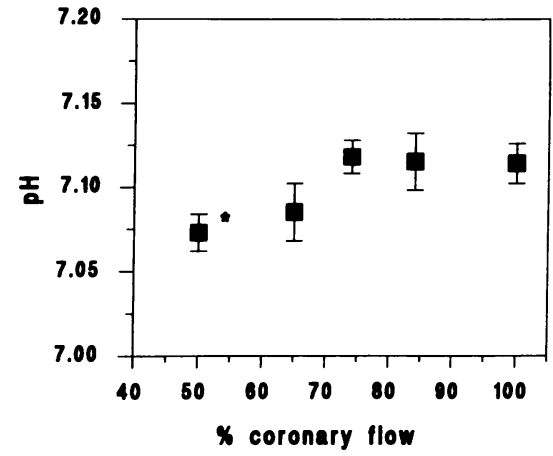

d

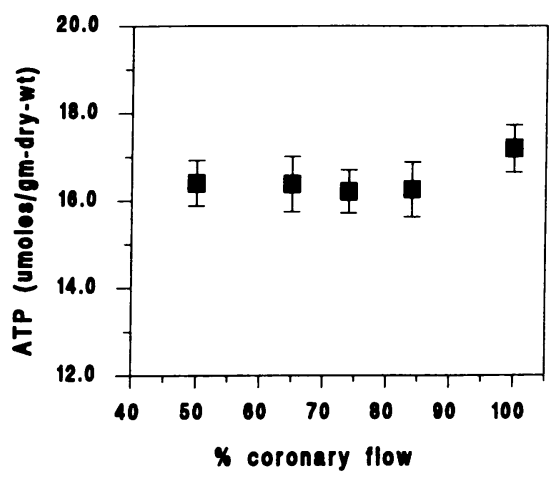

Figure 4. Coronary flow (\% of control) versus ${ }^{31} \mathrm{P}$-MRS-determined $(a)$ inorganic phosphate $\left(P_{i}\right),(b) \mathrm{pH},(c)$ phosphocreatine $(P C r)$, and $(d) \beta$-ATP from Langendorf-perfused rat hearts. Data are presented as mean \pm SEM for $n=11$ hearts. A significant increase of $P_{i}$ was observed when coronary flow was reduced to $75 \%$ of control. $\mathrm{PCr}$ and $\mathrm{pH}$ did not significantly decrease until coronary flow was reduced to $50 \%$ of control. ATP did not change at any coronary flow reduction studied. ${ }^{*} P$ $<0.05$. signal/noise ratio attained using our coil and experimental protocol, as well as the small, but consistent increase in $P_{i}$ that was observed during mild coronary flow reductions. Table II shows the pooled ${ }^{31} \mathrm{P}$-MRS data from the 10-min summed spectra. Fig. $4 a$ shows that $P_{i}$ increased as coronary flow was reduced, with a significant rise of $P_{i}$ observed when coronary flow was reduced to $75 \%$ of control, from $2.5 \pm 0.3$ to $4.2 \pm 0.4$ $\mu \mathrm{mol} / \mathrm{g}$ dry $\mathrm{wt}$. In contrast, $\mathrm{pH}$ did not significantly decrease until coronary flow was reduced $50 \%$, from $7.11 \pm 0.01$ to $7.07 \pm 0.01$ (Fig. 4 b). PCr did not significantly decrease until coronary flow was reduced to $50 \%$ of control, from $41.6 \pm 1.3$ to $38.1 \pm 1.5 \mu \mathrm{mol} / \mathrm{g}$ dry wt (Fig. $4 c$ ), and ATP was unchanged over the range of coronary flow reductions studied (Fig. $4 d$ ).

As shown in Fig. 5, $\mathrm{P}_{\mathrm{i}}$ demonstrated a close relationship with developed pressure, whereas other phosphate metabolites and $\mathrm{pH}$ did not (not shown). The $\mathrm{PCr} / \mathrm{P}_{\mathrm{i}}$ ratio demonstrated a linear relationship with coronary flow over the range of coronary flows studied, from $19.2 \pm 2.5$ to $5.3 \pm 0.6$ (Fig. $6 a$ ). $\Delta \mathrm{G}_{\mathrm{ATP}}$ also demonstrated a linear relationship with coronary flow from $-60.9 \pm 0.3$ to $-57.6 \pm 0.3 \mathrm{~kJ} / \mathrm{mol}$ (Fig. $6 \mathrm{~b}$ ).

To determine whether the increased $P_{i}$ was secondary to changes in energy phosphate metabolism resulting from ischemia versus an increase of unbound or NMR "visible" $P_{i}(20$, 24 ), energy phosphates ( $P C r, A T P, P_{i}$ ) were summed at baseline and coronary flow reductions. Although no significant change in total phosphate metabolites could be demonstrated, Table II shows an increasing trend with coronary flow reductions.

\section{Discussion}

The major findings of this study were $(a)$ during mild coronary flow reductions the cytosolic free calcium transient amplitude did not decrease, $(b)$ there was a close relationship between the rise of $P_{i}$ and the fall of developed pressure, with a statistically significant increase of $P_{i}$ when coronary flow was reduced to $75 \%$ of control; $(c)$ in contrast to $\mathrm{P}_{\mathrm{i}}, \mathrm{pH}$ was not altered until coronary flow was reduced to $50 \%$ of control, suggesting that $\mathrm{H}^{+}$does not play a major role in depressing contractility during mild coronary flow reductions, and $(d)$ further increases of vascular perfusion pressure above physiological control levels produced a further increase of developed pressure, with no

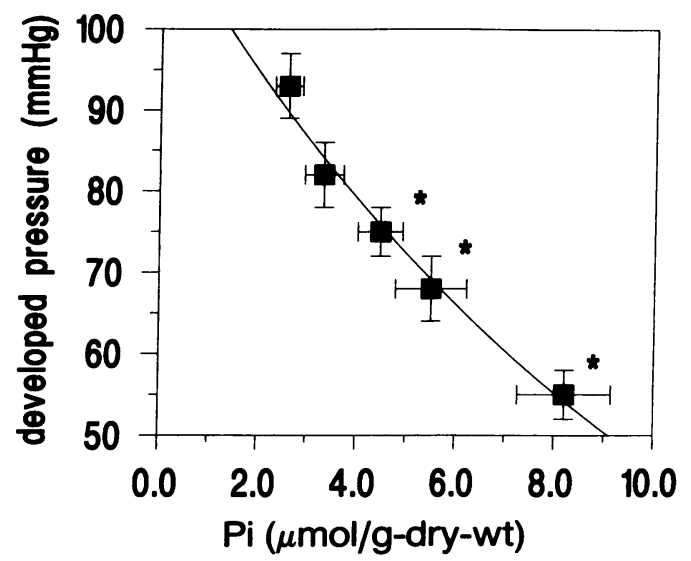

Figure 5. Relationship between inorganic phosphate $\left(P_{i}\right)$ and developed pressure $(\mathrm{mmHg})$ during mild coronary flow reductions in Langendorf-perfused rat hearts. Parameters were most closely correlated by an exponential fit $\left(r^{2}=0.98\right)$. A curvilinear regression was used to analyze the relationship between $P_{i}$ and developed pressure based on the assumption that this relationship is mediated through product inhibition of an enzymatic reaction. $r^{2}=0.96$ for a linear regression. ${ }^{*} P<0.05$. 


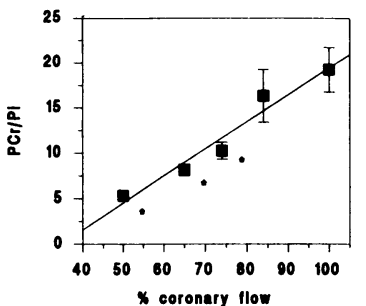

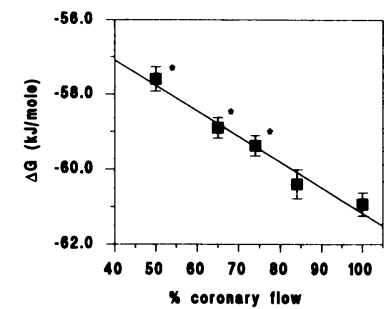

Figure 6. (a) The phosphocreatine/inorganic phosphate ratio $(\mathrm{PCr} /$ $\left.P_{i}\right)$ and $(b)$ the free energy change of ATP hydrolysis $\left(\Delta \mathrm{G}_{\mathrm{ATP}}\right)$ as a function of coronary flow (\% of control) in Langendorf-perfused rat hearts. Significant changes in both parameters were observed when coronary flow was reduced to $75 \%$ of control $\left(r^{2}=0.95\right.$ and 0.97 respectively). ${ }^{*} P<0.05$.

change of the cytosolic free calcium transient. These findings are consistent with the hypothesis that during mild reductions of coronary flow, myocardial contractile depression results from an altered relationship between cytosolic free calcium and pressure, not a decrease of the cytosolic free calcium transient amplitude. Additional experiments suggest that increased $\mathbf{P}_{\mathrm{i}}$ and decreased intravascular pressure, not $\mathrm{H}^{+}$, may mediate this alteration of the cytosolic free calcium-pressure relationship during mild reductions of coronary flow.

\section{Cytosolic free calcium transient}

A potential mediator of contractile depression during mild reductions of coronary flow is a decrease of the cytosolic free calcium transient. However, our findings demonstrate a significant decrease of contractility after mild coronary flow reductions in the absence of a decrease of the cytosolic free calcium transient. These data are in agreement with those of Lee et al. (1) using Indo-1 calcium fluorescence and Kihara et al. (2) using aequorin luminescence, who showed an increase of the cytosolic free calcium transient as contractility fell during acute no-flow ischemia.

In contrast, two other studies that examined the cytosolic free calcium transient during mild reductions in perfusion pressure demonstrated decreased $\Delta \mathrm{Ca}_{\mathbf{i}}(3,4)$. Wikman-Coffelt et al. (3), who measured $\Delta \mathrm{Ca}_{\mathrm{i}}$ on the right ventricular septal surface using Indo-1 calcium fluorescence, reported a $21 \%$ decrease in $\Delta \mathbf{C a}_{\mathrm{i}}$ when coronary perfusion pressure was reduced from 110 to $80 \mathrm{mmHg}$. The difference between these results and those of the present study may be due to differences in experimental design and Indo-1 fluorescence methods. Wikman-Coffelt et al. (3) monitored emission wavelengths that are not isosbestic with regard to alterations of tissue absorbance due to changes of the myoglobin oxygenation state $(16,17)$. Therefore, a change in the tissue filter effect after a decrease in perfusion pressure could cause a spurious change in $\Delta \mathrm{Ca}_{\mathrm{i}}$. Furthermore, Wikman-Coffelt et al. (3) used superphysiological coronary perfusion pressures and a lower bath calcium (1 $\mathrm{mM}$ ). This suggests that the conditions under which their measurements were made were less physiological than those of the present study.

Kitakaze and Marban (4), using the fluorine magnetic resonance spectroscopy calcium indicator 5F-BAPTA, demonstrated a decrease of $\Delta \mathrm{Ca}_{\mathrm{i}}$ of $>50 \%$ after a reduction of coronary perfusion pressure from 80 to $60 \mathrm{mmHg}$ (coronary flow reduced to $\sim 80 \%$ of control). However, their experimental conditions were very different from those of the present study.

A perfusate calcium concentration of $8 \mathrm{mM}$ was used to partially compensate for the calcium buffering effect of 5FBAPTA. Increasing perfusate calcium in the presence of 5FBAPTA results in developed pressures of $\sim 20-40 \mathrm{mmHg}$ and produces end diastolic pressures of $40-60 \mathrm{mmHg}$ (unpublished data from this laboratory). Furthermore, Kitakaze and Marban (4) observed a $>50 \%$ reduction of developed pressure when perfusion pressure was reduced by $25 \%$ compared with a fall of developed pressure of $<25 \%$ in hearts not perfused with 5F-BAPTA. This suggest that hearts loaded with 5F-BAPTA in the presence of high perfusate calcium may have an altered relationship between perfusion pressure and contractility. Finally, Kitakaze and Marban (4) used a reduced pacing rate of 1 $\mathrm{Hz}$ and a reduced perfusate temperature of $30^{\circ} \mathrm{C}$. Therefore, differences between the present study and previous studies are most likely due to differences in experimental design and methodology. Although no isolated perfused heart model can be considered truly physiological, the present study was designed to more closely mimic in vivo conditions (23).

A major criticism of cytosolic free calcium measurements obtained by Indo-1 calcium fluorescence in perfused hearts has been that data is obtained at the epicardial surface and, therefore, does not truly reflect transmural cytosolic free calcium levels after ischemic interventions. Transmural gradients of coronary flow and microvascular pressures have been demonstrated in porcine and canine hearts under normal physiological conditions and during low flow ischemia (25-27). However, significant differences in relative coronary flow or microvascular pressure in the endocardium compared with the epicardium did not occur with milder reductions in coronary perfusion pressure, only at more severe reductions. In the present study, parallel experiments performed at the endocardial and epicardial surfaces during coronary flow reductions to $50 \%$ of control demonstrated no significant change in the cytosolic free calcium transient amplitude or diastolic level. Therefore, the present observations at both the epicardium and endocardium most likely reflect cytosolic free calcium levels throughout the myocardium and suggest that myocardial contractility is not regulated by a decrease of the cytosolic free calcium transient during mild reductions of coronary flow.

\section{Potential mediators of contractile depression}

Inorganic phosphate. The observed increases of $\mathrm{P}_{\mathrm{i}}$ during mild coronary flow reductions (Fig. $4 a$ ) are in agreement with the in vivo work of Schaefer et al. (10) who demonstrated a significant fall of $\mathrm{PCr} / \mathrm{P}_{\mathrm{i}}$ at a coronary flow of $75 \%$ of control in an open-chest porcine model. Furthermore, observations by Path et al. (11) in an open-chest canine model, suggested a rise of endocardial $P_{i}$ after a reduction of coronary artery perfusion pressure to $\sim 70 \%$ of baseline. A recent study by Keller and Cannon (8) showed a relationship between decreases in coronary perfusion pressure and a fall of $\mathrm{PCr} / \mathrm{P}_{\mathrm{i}}$ in the perfused rat heart. However, several perfused heart studies have suggested that the fall in contractility associated with mild coronary flow reductions occurs without changes in cardiac energy phosphates $(6,7,14,15)$. Clarke and Willis (6) reported no change of energy phosphates until coronary flow was reduced to $\sim 30-42 \%$ of control but observed a change of the phosphorylation potential $\left(\mathrm{ATP} / \mathrm{ADP} \cdot \mathrm{P}_{\mathrm{i}}\right.$ ) at a coronary flow of $\sim 56 \%$ of control. Marshall (7), Weiss et al. (15), and Brooks et al. (14) reported no changes of energy phosphates until coronary flows of $\sim 30-33 \%$ of control. 
The present study, which demonstrates a significant increase of $P_{i}$ at a coronary flow to $75 \%$ of control, differs in several ways from the aforementioned reports. The signal/ noise ratio of the ${ }^{31} \mathrm{P}-\mathrm{MRS}$ spectra was substantially improved by acquiring data over 10 -min steady state periods of mild coronary flow reductions. The experimental protocol allowed for data acquisition at several coronary flow reductions produced in a random order, without the need to first produce "no-flow" ischemia, as was done in a previous study (6). Furthermore, coronary flow was returned to baseline between each flow reduction period. This prevented the possible complications of myocardial stunning or injury, as evidenced by the return of energy phosphate metabolites and mechanical function to baseline between interventions and at the end of each experiment. In addition, in the present study, other factors such as substrates, pacing rate, and coronary perfusion pressure more closely mimicked in vivo physiological conditions (23).

There are two possible explanations for the increase of $\mathbf{P}_{i}$ observed during mild coronary flow reductions. First, a decreased availability of oxygen may have impaired ATP synthesis by decreasing the rate of the creatine kinase reaction, leading to a rise of ADP and $P_{i}$. However, oxygen availability may not be limiting during mild coronary flow reductions. Jacobus et al. (5) found no reduction in tissue oxygen content, measured by mass spectrometry, until coronary flow was reduced to $48 \%$ of control in a perfused rabbit heart. The present finding that there was no change of $\mathrm{PCr}$ until coronary flow was reduced to $50 \%$ of control also suggests that ATP synthesis was not significantly limited by a decreased oxygen availability, at least at very mild flow reductions ( $75 \%$ of control), where $\mathbf{P}_{\mathbf{i}}$ had already significantly increased.

A second possibility that could account for the increase of $P_{i}$ associated with mild coronary flow reductions is that the amount of unbound or NMR visible $P_{i}$ may have increased during mild coronary flow reductions. Humphrey and Garlick (20) and Jeffry et al. (24) demonstrated in perfused rat hearts that the amount of NMR visible $P_{i}$ increased, relative to chemically determined $P_{i}$, during no-flow ischemia. This increase of NMR visible $P_{i}$ was attributed to the release of $P_{i}$ from binding sites on intracellular macromolecules after changes in the intracellular environment ( such as a change of pH) or a shift of $\mathbf{P}_{i}$ to the cytosol from an intracellular compartment (such as mitochondria) where $P_{i}$ cannot be detected by NMR. The present finding that total energy phosphates may have increased (although not a statistically significant increase) during graded reductions of coronary flow (Table II) supports the possibility that there was an increase in a NMR visible phosphate metabolite. Further studies will be necessary to elucidate the origin of the increased $P_{i}$ associated with mild coronary flow reductions.

The role of $P_{i}$ in depressing myocardial contractility during mild coronary flow reductions is supported by the findings of Kentish (12), who demonstrated that increases of $P_{i}$ caused a fall of contractile force in skinned myofibers. Kentish (12) further showed that a decrease in force by $P_{i}$ was the result of an altered calcium-force relationship; i.e., myofilament calcium sensitivity decreased $\left(\left[\mathrm{Ca}^{2+}\right]_{50}\right.$ rose) and the maximum calcium activated force decreased as $P_{i}$ was increased. An increase of bath $P_{i}$ from 1 to $2 \mathrm{mM}$ caused approximately a $20 \%$ decrease of force. In the present study, a similar relationship between increased $P_{i}$ and decreased developed pressure was observed. Assuming a cytosolic fraction of $0.44 \mathrm{ml} / \mathrm{g}$ wet weight
(21), an increase of $P_{i}$ from 0.9 to $2.0 \mathrm{mM}$ caused a $27 \%$ decrease in developed pressure. These similarities support the view that $P_{i}$ mediates contractile depression during mild coronary flow reductions by altering the calcium-pressure relationship.

Intravascular pressure. Another proposed mediator of contractile depression during mild coronary flow reductions is decreased intravascular pressure. The importance of the role of changes in intravascular pressure, the "garden hose" effect, in contractile depression during mild underperfusion remains controversial $(28,29)$. Some studies suggest that vascular collapse may be an important mediator of contractile depression after acute no-flow ischemia $(29,30)$. However, other studies have demonstrated a close relationship between the initial fall of pressure and energy phosphate metabolites after acute noflow ischemia, suggesting that vascular collapse is not important in mediating contractile depression $(31,32)$.

In the present study, although the role of decreased intravascular pressure was not directly examined, elevations of perfusion pressure $>71 \mathrm{mmHg}$ increased developed pressure further, without a change of cytosolic free calcium levels. This suggests that increased intravascular pressure may be an important mediator of the further increases of contractile force at higher perfusion pressures. Because of signal-to-noise resolution limitations, $P_{i}$ could not be assessed at these superphysiological pressures to determine if further decreases might occur.

Nevertheless, the finding of increased developed pressure at superphysiological perfusion pressures is consistent with the view that decreases in intravascular pressure during coronary flow reductions, as well as increased $P_{i}$, may play a role in mediating the contractile dysfunction through an alteration of the relationship between cytosolic free calcium and contractile force. Further studies will be necessary to determine the relative importance of vascular pressure as a mediator of contractile failure during graded reductions of coronary flow.

Intracellular acidosis. Intracellular acidosis has been considered another potential mediator of contractile depression during mild coronary flow reductions. The results of the present study are in agreement with the previous findings of Jacobus et al. (5), who also reported no change of intracellular $\mathrm{pH}$ until coronary flow was reduced to $50 \%$ of control. Additionally, other studies have demonstrated significant increases in lactate only when coronary flow was reduced below $50 \%$ of control $(8,15)$. Taken together with those of the present study, these data suggest that intracellular acidosis does not play a significant role in the contractile depression associated with mild coronary flow reductions.

\section{Conclusion}

The cytosolic free calcium transient amplitude did not decrease during mild reductions of coronary flow. These data support the hypothesis that during mild coronary flow reductions contractility is depressed by an alteration of the relationship between cytosolic free calcium and pressure, rather than by a decrease of the cytosolic free calcium transient. Furthermore, increased $P_{i}$ and decreased intravascular pressure may mediate this contractile depression by altering the cytosolic free calcium-pressure relationship. Intracellular acidosis does not appear to play a role in depressing contractility during mild coronary flow reductions. 


\section{Acknowledgments}

The authors thank Dr. Saul Schaefer for his many helpful discussions and review of the final manuscript.

This work was supported in part by National Institutes of Health Grants 1 F32 HL08244 (V. M. Figueredo), RO1 DK33923 (M. W. Weiner), 3 PO1 HL25847 (B. M. Massie), and K08 HL02448 (S. A. Camacho); American Heart Association, California Affiliate Grant-inAid 91-106 (S. A. Camacho); and the Research Service of the Department of Veterans Affairs (M. W. Weiner and B. M. Massie). This work was done during the tenure of a research fellowship from the American Heart Association, California Affiliate and with funds contributed by the Redwood Empire Chapter (R. Brandes).

\section{References}

1. Lee, H. C., R. Mohabir, N. Smith, M. R. Franz, and W. T. Clusin. 1988 Effect of ischemia on calcium-dependent fluorescence transients in rabbit hearts containing Indo 1 . Correlation with monophasic action potentials and contraction. Circulation. 78:1947-1959.

2. Kihara, Y., W. Grossman, and J. P. Morgan. 1989. Direct measurement of changes in intracellular calcium transients during hypoxia, ischemia, and reperfusion of the intact mammalian heart. Circ. Res. 65:1029-1044.

3. Wikman-Coffelt, J., S. T. Wu, and W. W. Parmley. 1991. Intracellular endocardial calcium and myocardial function in rat hearts. Cell Calcium. 12:3950 .

4. Kitakaze, M., and E. Marban. 1989. Cellular mechanism of the modulation of contractile function by coronary perfusion pressure in ferret hearts. J. Physiol. 414:455-472.

5. Jacobus, W. E., I. H. Pores, S. K. Lucas, M. L. Weisfeldt, and J. T. Flaherty. 1982. Intracellular acidosis and contractility in the normal and ischemic heart as examined by ${ }^{31}$ P NMR. J. Mol. Cell. Cardiol. 14(3):13-20.

6. Clarke, K., and R. J. Willis. 1987. Energy metabolism and contractile function in rat heart during graded, isovolumic perfusion using ${ }^{31} \mathrm{P}$ nuclear magnetic resonance spectroscopy. J. Mol. Cell. Cardiol. 19:1153-1160.

7. Marshall, R. C. 1988. Correlation of contractile dysfunction with oxidative energy production and tissue high energy phosphate stores during partial coronary flow disruption in rabbit heart. J. Clin. Invest. 82:86-95.

8. Keller, A. M., and P. J. Cannon. 1991. Effect of graded reductions of coronary pressure and flow on myocardial metabolism and performance: a model of "hibernating" myocardium. J. Am. Coll. Cardiol. 17:1661-1670.

9. Lavanchy, N., J. Martin, and A. Rossi. 1984. Graded global ischaemia and reperfusion of the isolated perfused rat heart: characterisation by ${ }^{31} \mathrm{P}$ NMR spectroscopy of the extent of energy metabolism damage. Cardiovasc. Res. 18:573582.

10. Schaefer, S., G. Schwartz, J. R. Gober, A. K. Wong, S. A. Camacho, B. Massie, and M. W. Weiner. 1990. Relationship between myocardial metabolites and contractile abnormalities during graded regional ischemia ${ }^{31} \mathbf{P}$ NMR studies of porcine myocardium in vivo. J. Clin. Invest. 85:706-713.

11. Path, G., P. M. Robitaille, H. Merkle, M. Tristani, J. Zhang, M. Garwood, A. H. L. From, R. J. Bache, and K. Ugurbil. 1990. Correlation between transmural high energy phosphate levels and myocardial blood flow in the presence of graded coronary stenosis. Circ. Res. 67:660-673.

12. Kentish, J. C. 1986. The effects of inorganic phosphate and creatine phosphate on force production in skinned muscles from rat ventricle. J. Physiol. 370:585-604

13. Godt, R. E., and T. M. Nosek. 1989. Changes of intracellular milieu with fatigue or hypoxia depress contraction of skinned rabbit skeletal and cardiac muscle. J. Physiol. 412:155-180.

14. Brooks, W. M., L. J. Haseler, K. Clarke, and R. J. Willis. 1986. Relation between the phosphocreatine to ATP ratio determined by ${ }^{31} \mathrm{P}$ nuclear magnetic resonance spectroscopy and left ventricular function in underperfused guinea-pig heart. J. Mol. Cell. Cardiol. 18:149-155.

15. Weiss, R. G., V. P. Chacko, J. D. Glickson, and G. Gerstenblith. 1989. Comparative ${ }^{13} \mathrm{C}$ and ${ }^{31} \mathrm{P}$ NMR assessment of altered metabolism during graded reductions in coronary flow in intact rat hearts. Proc. Natl. Acad. Sci. USA. 86:6426-6430.

16. Brandes, R., V. M. Figueredo, S. A. Camacho, and W. M. Weiner. 1991 $\mathrm{Ca}^{2+}$ measurements in perfused hearts by fluorescence. Effects of motion, tissue absorbance and NADH. Circulation. 84(4):II-169a. (Abstr.)

17. Fralix, T. A., F. W. Heineman, and R. S. Balaban. 1990. Effects of tissue absorbance on NAD $(P) H$ and Indo-1 fluorescence from perfused rabbit hearts. FEBS (Fed. Eur. Biochem. Soc.) Lett. 262:287-292.

18. Lorell, B. H., C. S. Apstein, M. J. Cunningham, F. J. Schoen, E. O. Weinberg, G. A. Peeters, and W. H. Barry. 1990. Contribution of endothelial cells to calcium-dependent fluorescence transients in rabbit hearts loaded with Indo-1. Circ. Res. 67:415-425.

19. van der Veen, J. W. C., R. de Beer, P. R. Luyten, and D. Van Ormondt 1988. Accurate quantification of in vivo ${ }^{31} \mathrm{P}$ NMR signals using the variable projection method and prior knowledge. Magn. Reson. Med. 6:92-98.

20. Humphrey, S. M., and P. B. Garlick. 1991. NMR-visible ATP and $P_{i}$ in normoxic and reperfused rat hearts: a quantitative study. Am. J. Physiol. 29:H6H12.

21. Matthews, P. M., D. J. Taylor, and G. K. Radda. 1986. Biochemical mechanisms of acute contractile failure in the hypoxic rat heart. Cardiovasc. Res. 20:13-19.

22. Kammermeier, H., P. Schmidt, and E. Jungling. 1982. Free energy change of ATP-hydrolysis: a causal factor of early hypoxic failure of the myocardium? $J$. Mol. Cell. Cardiol. 14:267-277.

23. Bittl, J. A., J. A. Balschi, and J. S. Ingwall. 1987. Contractile failure and high-energy phosphate turnover during hypoxia: ${ }^{31} \mathrm{P}-\mathrm{NMR}$ surface coil studies in living rat. Circ. Res. 60:871-878.

24. Jeffry, F. M., C. J. Storey, R. L. Nunnally, and C. R. Malloy. 1989. Effect of ischemia on NMR detection of phosphorylated metabolites in the intact rat heart. Biochemistry. 28:5323-5326.

25. Weintraub, W. S., S. Hattori, J. B. Agarwal, M. M. Bodenheimer, V. S Banka, and R. H. Helfant. 1981. The relationship between myocardial blood flow and contraction by myocardial layer in the canine left ventricle during ischemia. Circ. Res. 48:430-438.

26. Chilian, W. M. 1991. Microvascular pressures and resistances in the left ventricular subepicardium and subendocardium. Circ. Res. 69:561-570.

27. Griggs, D. M., and Y. Nakamura. 1968. Effect of coronary constriction on myocardial distribution of iodoantipyrine- ${ }^{131}$ I. Am. J. Physiol. 215(5):10821088.

28. Arnold, G., F. Kosche, E. Miessner, A. Neitzert, and W. Lochner. 1968. The importance of the perfusion pressure in the coronary arteries for the contractility and the oxygen consumption of the heart. Pfluegers Arch. Eur. J. Physiol. 299:339-356.

29. Feigl, E. O. 1983. Coronary physiology. Physiol. Rev. 63(1):1-205.

30. Koretsune, Y., M. C. Corretti, H. Kusuoka, and E. Marban. 1991. Mechanism of early ischemic contractile failure; inexcitability, metabolite accumulation, or vascular collapse? Circ. Res. 68:255-262.

31. Schwartz, G. G., S. Schaefer, D. J. Meyerhoff, J. Gober, P. Fochler, B Massie, and M. W. Weiner. 1990. Dynamic relation between myocardial contractility and energy metabolism during and following brief coronary occlusion in the pig. Circ. Res. 67:490-500.

32. Clarke, K., A. J. O'Conner, and R. J. Willis. 1987. Temporal relation between energy metabolism and myocardial function during ischemia and reperfusion. Am. J. Physiol. 253:H412-H421. 